\title{
Amygdala response to self-critical stimuli and symptom improvement in psychotherapy for depression
}

Nadja Doerig, Tobias Krieger, David Altenstein, Yolanda Schlumpf, Simona Spinelli, Jakub Späti, Janis Brakowski, Boris B. Quednow, Erich Seifritz and Martin grosse Holtforth

\section{Background}

Cognitive-behavioural therapy is efficacious in the treatment of major depressive disorder but response rates are still far from satisfactory.

\begin{abstract}
Aims
To better understand brain responses to individualised emotional stimuli and their association with outcome, to enhance treatment.
\end{abstract}

\section{Method}

Functional magnetic resonance imaging data were collected prior to individual psychotherapy. Differences in brain activity during passive viewing of individualised self-critical material in 23 unmedicated out-patients with depression and 28 healthy controls were assessed. The associations between brain activity, cognitive and emotional change, and outcome were analysed in 21 patients.

\section{Results}

Patients showed enhanced activity in the amygdala and ventral striatum compared with the control group. Nonresponse to therapy was associated with enhanced activity in the right amygdala compared with those who responded, and activity in this region was negatively associated with outcome. Emotional but not cognitive changes mediated this association.

\section{Conclusions}

Amygdala hyperactivity may lessen symptom improvement in psychotherapy for depression through attenuating emotional skill acquisition.

\section{Declaration of interest}

None.

\section{Copyright and usage}

(c) The Royal College of Psychiatrists 2016.
Harsh self-criticism not only triggers strong negative emotional responses but also is a symptom of depression itself. Accordingly, pervasive self-critical thinking is considered to be a risk factor for the development and maintenance of depression, ${ }^{1}$ and is consequently an important psychotherapeutic target. ${ }^{2}$ Cognitive-behavioural therapy (CBT) is an empirically supported intervention that is efficacious in the acute treatment of major depressive disorder in $40-60 \%$ of patients. Hence, depending on patient and therapy characteristics, a large proportion of patients still fail to achieve an adequate response.

The identification of neural abnormalities in depression during self-critical processing and the search for related neurophysiological markers of outcome prediction might advance differential treatment selection. In this study we examine the potential psychological mechanisms linking neural markers to psychotherapy outcomes. This integrative neurophysiologicalpsychological strategy might help to optimise individualised treatment strategies for patients with depression. Major depressive disorder is characterised by disturbances in emotional processing. In processing emotional stimuli, the amygdala functions by mediating attention, assigning an evaluation to the emotional stimulus and storing emotionally significant events in long-term memory. Additionally, the amygdala has numerous connections with other brain regions, such as the ventral striatum, which further process and integrate emotional information. ${ }^{3}$ Previous studies have reported depression-related hyperactivity in the amygdala as well as in the ventral striatum, ${ }^{4,5-9}$ probably causing negatively biased emotion processing, for instance in the evaluation and judgement of emotional stimuli. The majority of related functional magnetic resonance imaging (fMRI) studies have used standardised emotional pictures, masked or unmasked facial stimuli or personally relevant rating tasks for examining neural responses to emotional stimuli. However, using standardised emotional paradigms, several authors also reported no difference in amygdala activity between patients with depression and a control group (see Townsend et al for an overview). ${ }^{10}$ In contrast, the few studies using specific and personally relevant emotional stimuli have reported robust enhanced activation of striatal regions and the amygdala in people with acute depression, ${ }^{8,11,12}$ as well as in patients with a history of depression confronted with critical comments about their own mothers. ${ }^{13}$ Moreover, passive processing of emotional visual stimuli is associated with a higher probability of amygdala activation than processing accompanied by active task instructions. ${ }^{14}$ A previous study has shown enhanced amygdala activity during confrontation with individualised self-critical stimuli compared with neutral stimuli in a healthy sample. ${ }^{15}$ Accordingly, methodological heterogeneity regarding individualisation of stimuli and mode of presentation may explain some of the mixed findings, in addition to sample differences in symptom profiles, medication status, levels of depression severity or comorbid anxiety disorders. ${ }^{10}$ Consequently, the method chosen in our study - examining abnormal responses of the amygdala and ventral striatum to individualised, painful self- critical stimuli in an unmedicated patient sample with depression using the technique of passive exposure to stimuli promises to yield more ecologically valid and robust results.

Functional magnetic resonance imaging assessment has been suggested as a powerful strategy for identifying prognostic markers of clinical response. ${ }^{16}$ Using a meta-analytic approach, Fu et al found enhanced activity in the right striatum and anterior insula to be associated with a lower likelihood of clinical benefit from treatment including CBT and medication. ${ }^{16}$ However, previous studies of amygdala response have yielded heterogeneous results regarding outcome prediction. Related findings have 
indicated increased baseline amygdala activation in those who subsequently responded to therapy but have also shown the reverse relationship, with increased amygdala activity associated with poor outcomes during the treatment of depression and post-traumatic stress disorder (PTSD). ${ }^{17}$ Moreover, several studies have reported no significant relationship between amygdala activity and outcome (see Fu et al for an overview). ${ }^{16}$

In accordance with the results of studies using individualised emotional stimuli, we predicted enhanced response in the amygdala and ventral striatum in patients with depression compared with a matched healthy control group. In addition, we explored differences between those who did and did not respond to therapy, as well as the potential prediction of treatment outcome by differences in amygdala and striatal activity. If such predictions were verified we aimed to explore the associated psychological change mechanisms, defined as therapeutic processes that have the potential to ameliorate psychopathological disorder. ${ }^{18}$ Among other factors, change in dysfunctional cognition as well as the acquisition of emotional skills have been identified as psychological change mechanisms that are associated with better CBT outcomes. ${ }^{19,20}$ Consequently, we set out to examine whether the potentially identified biomarker-outcome relationships would be mediated by changes in dysfunctional cognitions and/or by acquisition of emotional skills.

\section{Method}

We investigated a sample of patients with a current major depressive episode (MDE; $n=23$ ) and a control group of healthy individuals $(n=28)$. All participants were right-handed, native German speakers and showed no contraindication to MRI. The study procedure was approved by the local ethics committee, and all participants provided advance written informed consent. Treatment was free of charge, and participation in the fMRI assessment was reimbursed according to local standards. Patients were recruited from a larger study of cognitive-emotional processing in psychotherapy. The fMRI data of six control group participants had been analysed for a previous publication. ${ }^{15}$

To qualify for the study, patients were required to have a minimum score of 14 on the Beck Depression Inventory II (BDI-II) at screening. ${ }^{21}$ Average depression severity at baseline was moderate, with a mean BDI-II score of 26.6 (s.d.=9.3). Diagnoses of MDE were confirmed with the Structured Clinical Interview for DSM-IV. ${ }^{22}$ Exclusion criteria included a history of mania or psychotic symptoms; borderline, antisocial or schizotypal personality disorder; and current substance dependence. In total, 24 persons with major depressive disorder were scanned. One person was excluded from further study owing to lack of motivation and strong discomfort during the scan. Thus, the data for 23 patients ( 11 women; mean age 37.4 years, s.d. $=17.1$ ) were included. Most of the patients (70\%) were diagnosed with a recurrent MDE (1 patient with one previous episode, 11 patients with two to five episodes and 3 patients with more than five previous episodes; 1 missing data), whereas the remaining 30\% met criteria for a first MDE. Other comorbid diagnoses, including anxiety disorders (39\%), were acceptable as long as depression was the primary treatment focus. The patients did not take any psychotropic medication for at least 4 weeks prior to the scan sessions and remained medication-free throughout the treatment and 3-month follow-up period. Fifteen patients were medicationnaïve, and six patients had taken selective serotonin or serotoninnoradrenaline reuptake inhibitors before the study ( 5 patients stopped more than 4 years before the study began, 1 patient stopped 1 year before). Additionally, one patient took a tricyclic antidepressant (stopped 1 year before the study) and for one patient we have no further information about medication intake prior to the study beyond the required 4 weeks. Healthy participants in the control group had no personal or first-degree relative history of major depressive disorder and no self-reported psychiatric problems ( 13 women; mean age 35.6 years, s.d. $=12.6$ ). The two study groups did not differ in age $(P=0.60)$, gender distribution $(P=0.92)$, marital status $(P=0.44)$ or highest level of education $(P=0.17)$.

After comparing the neural activity of the healthy participants with that of the participants with depression, we further compared brain activity at baseline between those who did or did not respond to CBT, and studied eventual links with therapy outcomes. Two patients withdrew before completing the 22 therapy sessions (sessions 15 and 19) and were not included in the longitudinal analysis. The resulting patient sample comprised 21 participants (10 women; mean age 38.3 years, s.d. $=12.4$ ). Another three patients had missing data at follow-up.

\section{Psychometric measures}

Symptom severity in the group with depression was measured using the German version of the 21 -item BDI-II. ${ }^{21,23}$ For the assessment of emotion regulation skills we used the total score of the 27-item German version of the Emotion Regulation Skills Questionnaire (ERSQ). ${ }^{24}$ Dysfunctional cognitions were assessed using the 40-item German version of the Dysfunctional Attitude Scale (DAS). ${ }^{25}$ To control for trait anxiety we used the 20 -item trait form of the State-Trait Anxiety Inventory (STAI). ${ }^{26}$

\section{Procedure}

Psychotherapy was offered as a research therapy separate from routine care in the out-patient clinic of the University of Zurich's psychology department. In total, 15 different therapists ( 2 men, 13 women) conducted the treatments, each treating one or two patients. All patients were scanned before the onset of their 22 weekly individual CBT sessions. Depressive symptoms were assessed before therapy at baseline (pre-treatment), directly after therapy ended (post-treatment) and 3 months later (follow-up). We defined response to treatment a priori as a minimum pre- to post-treatment reduction of $50 \%$ in BDI-II scores, on the basis of a previous study comparing pre-treatment brain response to fearful and neutral facial expressions and response to CBT in PTSD. ${ }^{17}$ Furthermore, a validation study of response and remission criteria reported that response best corresponded to a BDI change of $47 \%$, showing a sensitivity of $80 \%$ and a specificity of $67 \% .{ }^{27}$ Moreover, among self-report measures, the BDI-II has achieved wide acceptance and is sensitive to change. ${ }^{23}$ Assessments with the ERSQ and the DAS took place both immediately prior to and after treatment. Trait anxiety was assessed at baseline.

\section{Stimuli and task}

During the fMRI measurement, participants were presented with three different types of adjectives in six $29 \mathrm{~s}$ blocks of four adjectives each: neutral adjectives, individualised self-critical adjectives and individualised negative adjectives that were not self-critical. Furthermore, six $29 \mathrm{~s}$ blocks of rest (looking at a fixation cross) were inserted randomly. Each block contained an introduction indicating the condition (neutral, negative, selfcritical) followed by the presentation of the four adjectives and a request to press a button. In this paper we show only analyses of the neutral and self-critical conditions. Figure 1 shows the presentation times for a self-critical and a neutral block. For each person we ran one session lasting approximately $12 \mathrm{~min}$. The 

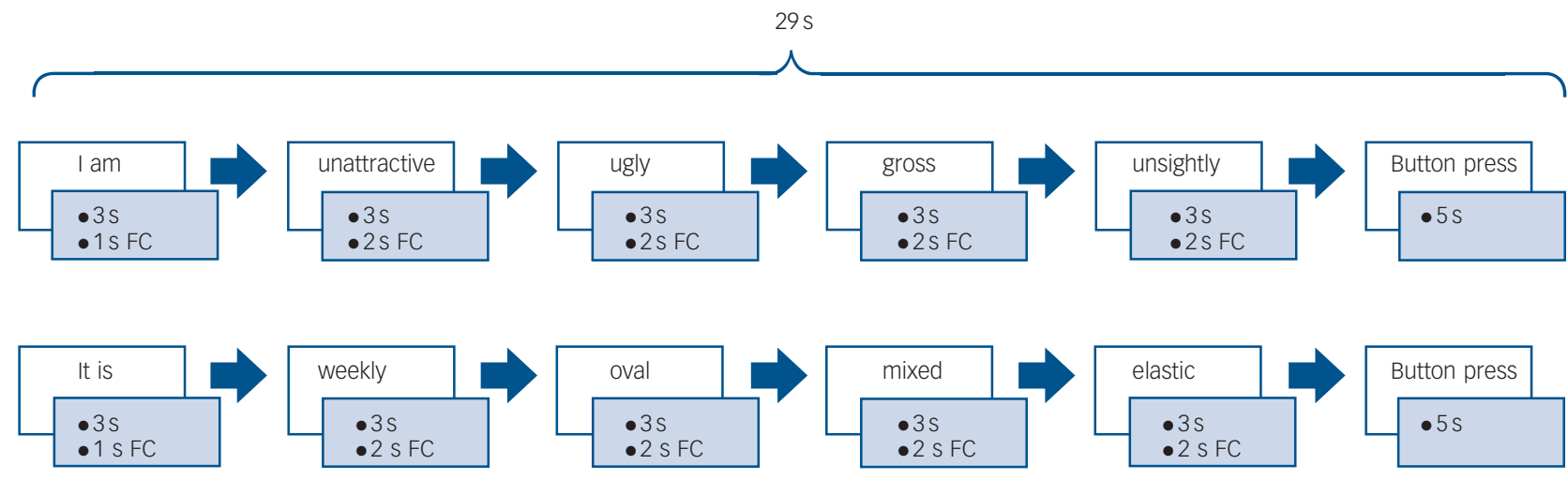

Fig. 1 Example of a self-critical and a neutral block including presentation times in seconds for a participant choosing the prototype 'unattractive' as self-critical. FC, fixation cross.

order of presentation was completely randomised and differed between participants. Neutral adjectives were the same for all participants and were pre-selected by the authors from 100 potentially neutral words. Forty-two volunteers $(21$ women and 21 men) rated the valence of all adjectives using a scale ranging from -2 (very negative) to +2 (very positive). Twenty-four adjectives were selected with modal values of 0 and mean values between -0.2 and 0.2 . Individualised self-critical and negative non-self-critical blocks consisted of prototypes (e.g. fat, boring, jealous) that participants chose individually from a list of 52 negative attributes. During the imaging experiment prototypes were presented interspersed with their three respective synonyms that were chosen based on a German word database (http:// wortschatz.uni-leipzig.de) and assumed to activate the same selfschema. All stimuli consisted of one to three syllables and at least 12 letters. We made sure that all adjectives reached a frequency level of at least 20 according to the Leipzig Word Database (frequency level based on Zipf's law, which states that the reference word 'the' ('der') is $2 \wedge 20$ times more frequent than the respective word, ensuring common use in everyday language). Participants were instructed to read the adjectives silently and focus on their meaning as well as on the triggered emotional reactions. For more details about the stimuli and their assessment see our previous study. ${ }^{15}$

\section{Imaging}

The fMRI scanning took place at a university psychiatric hospital in Switzerland using a $3 \mathrm{~T}$ Philips Intera whole-body MR unit equipped with an 8-channel Philips sensitivity-encoding (SENSE) head coil (www.healthcare.philips.com). Functional time series were acquired with a SENSE single-shot echoplanar imaging sequence. $^{28}$ Thirty-six contiguous axial slices were placed along the anterior-posterior commissure plane covering the entire brain. A total of $247 T_{2}{ }^{*}$-weighted echoplanar image volumes with blood oxygen level-dependent (BOLD) contrast (repetition time $3000 \mathrm{~ms}$, echo time $35 \mathrm{~ms}, 8080$ voxel matrix, interpolated to $128 \times 128$, voxel size $2.75 \times 2.75 \times 4 \mathrm{~mm}^{3}$, SENSE acceleration factor $R=2.0$ ) were acquired. The first four scans were discarded owing to $T_{1}$ saturation effects. For each participant a $T_{1}$-weighted high-resolution image was acquired.

\section{Data preprocessing and statistical analysis}

The functional image data were preprocessed and analysed in SPM8 (www.fil.ion.ucl.uk/spm) and implemented in MATLAB R2011a (Mathworks, Natick, Massachusetts, USA) using standard preprocessing steps. $^{29}$ First-level analysis (fixed effects) was performed on each participant's data including the six movement regressors and the three condition regressors. The BOLD data were modelled with a block design convolved with the standardised canonical haemodynamic response function and its temporal derivative. Estimated beta parameters and $t$-contrast images were brought to the second-level analysis (random effects). For both fMRI data group analyses (patients $v$. controls; response $v$. nonresponse), images of the contrast of interest (self-critical $v$. neutral) were analysed using two-sample $t$-tests. A voxel-wise threshold of $P<0.001$, requiring more than 10 contiguous voxels, was applied. According to our a priori hypotheses, we reported amygdala and striatal structures significant at $P<0.05$ family-wise error corrected at the cluster level after small volume correction (SVC) in SPM using the bilateral anatomical masks created with the WFU Pickatlas toolbox (www.fil.ion.ucl.ac.uk/spm/ext/). All coordinates are reported in Montreal Neurological Institute (MNI) space and peak activations were labelled according to the Anatomical Automated Labeling atlas implemented in SPM (www.fil.ion.ucl.ac.uk/spm/ext/). Analyses of sociodemographic data $\left(\chi^{2}\right.$-tests) were performed to ascertain comparability of the two respective groups. Furthermore, as structural volume differences of the amygdala might affect the findings of the cross-sectional analyses, we further ran SVC analysis integrating grey-matter volumes for each participant as a covariate vector of the left and right amygdala separately (see online supplement DS1 for the structural MRI data acquisition and voxel-based morphometry).

To examine change in depressive symptoms independent of initial severity, residual gain scores were calculated from a regression of pre-treatment BDI-II scores on post-treatment and follow-up scores. ${ }^{17}$ Associations of possible predictors and outcome were analysed using Pearson correlations (two-tailed) between the BOLD signal extracted from $6 \mathrm{~mm}$ radius spheres around peak voxels from the responder-analysis and residual BDI-II gain scores at post-treatment and follow-up. Mean beta parameter estimates were extracted using a MATLAB script programmed in-house.

Mediation analyses were calculated using the PROCESS script by Preacher \& Hayes. ${ }^{30}$ The total effect $(c)$ of an independent variable on a dependent variable is composed of a direct effect $\left(c^{\prime}\right)$ and an indirect effect through a proposed mediator. The indirect effect was computed by bootstrapping re-sampling with 1000 samples. We considered point estimates of the indirect effects as significant in case zero was not included in the $95 \%$ confidence interval. We used residual BDI-II gain scores at post-treatment and follow-up as dependent variables. Potential mediator variables were residual DAS and ERSQ gain scores, reflecting changes in 
cognitive and emotional processes during therapy. Correlation and mediation analyses were performed in PASW Statistics version 18.0 (IBM, Switzerland).

\section{Results}

\section{Cross-sectional analyses}

In accordance with our hypotheses, participants with major depressive disorder showed enhanced activity in the left amygdala (coordinates: $-22,-6,-16 ; t_{49}=4.05, \kappa=21$ ), the bilateral putamen (right: coordinates $36,0,-4 ; t_{49}=4.13, \kappa=31$; left: coordinates $\left.-32,-10,4 ; t_{49}=3.93, \kappa=20\right)$ and two clusters in the right caudate nucleus (coordinates $6,12,-2 ; t_{49}=4.63$, $\kappa=80$; coordinates $20,26,0 ; t_{49}=4.09, \kappa=14$ ), surviving SVC at $P<0.05$. Significant voxels in the right amygdala (coordinates 30 , $\left.2,-16 ; t_{49}=3.62, \kappa=4\right)$ did not reach our predefined cluster size $(\kappa>10)$. Moreover, separate SCV analyses for each hemisphere including the mean amygdala grey matter volumes of the respective hemispheres as covariates were performed. As described in online supplement DS1, we had to exclude three healthy individuals from these analyses because of the poor quality of the structural data. Small volume correction analyses resulted in significant differences for the left $(t=4.74, P=0.004, \kappa=54)$ and right hemispheres $(t=4.16, P=0.014, \kappa=13)$. Cortical group differences at the whole-brain level are reported in online Table DS1.

\section{Prediction analyses}

\section{Clinical response}

We divided those who completed therapy $(n=21)$ into two groups: those who responded to treatment $(n=13)$ and those who did not $(n=8)$ (Table 1$)$. Figure 2 shows the distribution of the patients as well as assignments to the respective groups. At baseline there was no statistically significant difference between the two groups regarding gender distribution $(P=0.86)$, age $(P=$ $0.98)$, marital status $(P=0.63)$, highest educational level $(P=0.22)$, single or recurrent episodes $(P=0.75)$, chronicity $(P=0.92)$ or comorbid anxiety disorder $(P=0.60)$. There was no significant difference on any of the assessed measures at baseline between the groups, but there were significant differences at post-treatment for the BDI-II and the ERSQ (Table 1).

\section{Differences in brain activity}

The non-responder and responder groups differed only in the right amygdala (coordinates 26, $2,-20 ; t_{19}=4.30, \kappa=19$ ), with the non-responder group showing enhanced activity, surviving SVC at $P>0.05$ (Fig. 2(a,b)). Because amygdala activity might be related to depression severity or to anxiety, ${ }^{31,32}$ we included in a further step BDI-II pre-treatment score, trait anxiety or comorbid anxiety as covariates of interest in the between-group analysis. This affected the results only slightly (online Table DS2). No difference in the activation of striatal regions was detected. Moreover, the responder group did not show higher activity in any region compared with the non-responder group.

\section{Baseline neural activity and treatment response}

The BOLD response in the right amygdala during confrontation with self-critical material correlated positively with residual BDI-II gain scores at post-treatment $(n=21 ; r=0.52, P=0.02)$ (Fig. 2(c)) and follow-up $(n=18 ; r=0.53, P=0.02)$. Therefore, poor response to CBT was significantly associated with increased right amygdala recruitment during the processing of self-critical material.
Table 1 Psychometric data for the sample before receiving cognitive-behavioural therapy and at post-treatment and 3-month follow-up

\begin{tabular}{|c|c|c|c|}
\hline & \multicolumn{2}{|c|}{ Score, mean (s.d.) } & \multirow[b]{2}{*}{$P$} \\
\hline & $\begin{array}{l}\text { Responder group } \\
(n=13)\end{array}$ & $\begin{array}{l}\text { Non-responder group } \\
\qquad(n=8)\end{array}$ & \\
\hline \multicolumn{4}{|l|}{ BDI-II } \\
\hline Pre-treatment & $27.85(8.44)$ & 26.13 (10.39) & 0.68 \\
\hline Post-treatment & $6.23(5.54)$ & 21.25 (7.19) & $<0.001$ \\
\hline Follow-up & $8.30(7.62)^{\mathrm{a}}$ & $19.75(15.00)$ & 0.05 \\
\hline \multicolumn{4}{|l|}{ ERSQ } \\
\hline Pre-treatment & 79.36 (12.38) & 71.13 (13.30) & 0.16 \\
\hline Post-treatment & $109.12(12.54)$ & $75.88(14.97)$ & $<0.001$ \\
\hline \multicolumn{4}{|l|}{ DAS } \\
\hline Pre-treatment & $148.36(24.13)$ & 131.25 (31.04) & 0.17 \\
\hline Post-treatment & $128.64(28.60)^{\mathrm{b}}$ & $137.20(23.81)$ & 0.50 \\
\hline \multicolumn{4}{|l|}{ STAI Trait } \\
\hline Pre-treatment & $60.01(9.62)$ & $56.131(12.63)$ & 0.42 \\
\hline \multicolumn{4}{|c|}{$\begin{array}{l}\text { BDI-II, Beck Depression Inventory II; DAS, Dysfunctional Attitude Scale; ERSQ, Emotion } \\
\text { Regulation Skills Questionnaire; STAl, State-Trait Anxiety Inventory. } \\
\text { a. } n=10 \text {. } \\
\text { b. } n=12 \text {. }\end{array}$} \\
\hline
\end{tabular}

\section{Mediation analysis}

To explain the association between amygdala activity and negative outcome in CBT, we evaluated the change in dysfunctional cognition and in emotional skills as theoretically derived and empirically supported mechanisms of change that might be compromised by amygdala hyperactivity. In the related mediation analysis, the association between right amygdala activity before therapy and residualised depression scores post-therapy was mediated by emotional skill acquisition. After controlling for this acquisition the relation between amygdala activity and outcome was no longer significant, indicating full mediation (Table 2). This effect survived introduction of comorbid anxiety and/or trait anxiety as covariates. Furthermore, we found similar mediation effects when the residual BDI-II gain score at follow-up was the dependent variable $(n=18)$. The replacement of missing data at follow-up using a last observation carried forward approach by integrating post-treatment measurement data for three patients revealed comparable effects (online Table DS3). In contrast, there was no mediation by change of dysfunctional attitudes at posttreatment and follow-up (Table 2). Online Fig. DS1 shows the mediation path model.

\section{Discussion}

We examined the role of amygdala and striatal response after confrontation with individualised self-critical stimuli in unmedicated patients with depression and healthy control participants. Additionally, the potential of these subcortical activations as biomarkers of CBT outcome prediction in concert with likely psychological change mechanisms was investigated.

\section{Patients $\boldsymbol{v}$. healthy participants}

In accordance with our hypothesis and with previous findings, patients showed enhanced activity compared with control participants in the amygdala and the bilateral ventral striatum (bilateral putamen and caudate nucleus) after emotional activation, which potentially indicates an altered neural circuitry involved in emotion processing. ${ }^{3,8}$ Our results therefore further support the use of more ecologically valid task designs using individualised stimuli for the study of abnormal subcortical 
(a)

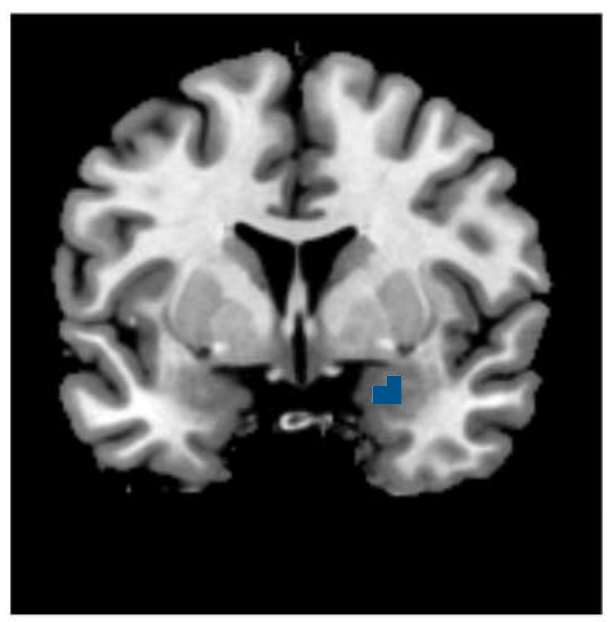

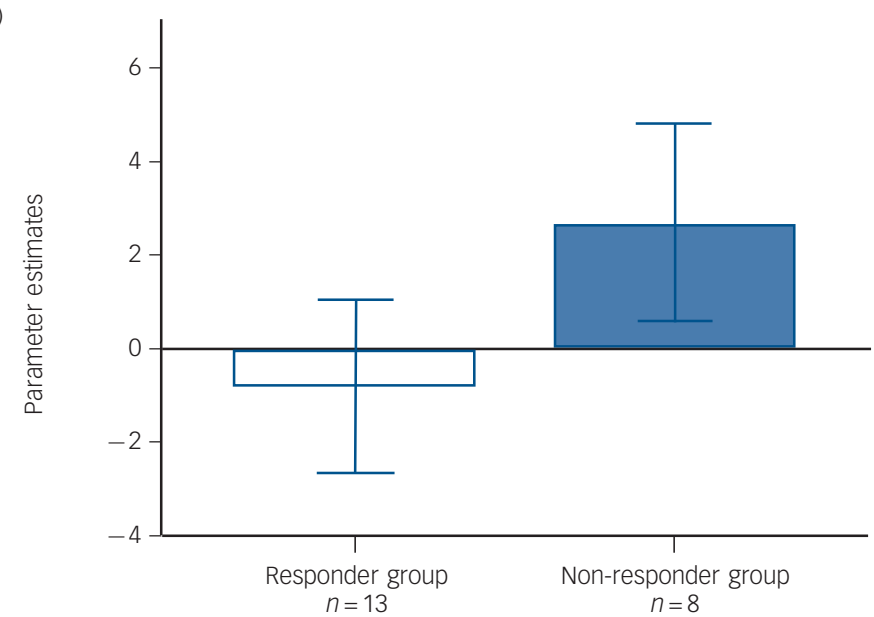

(C)

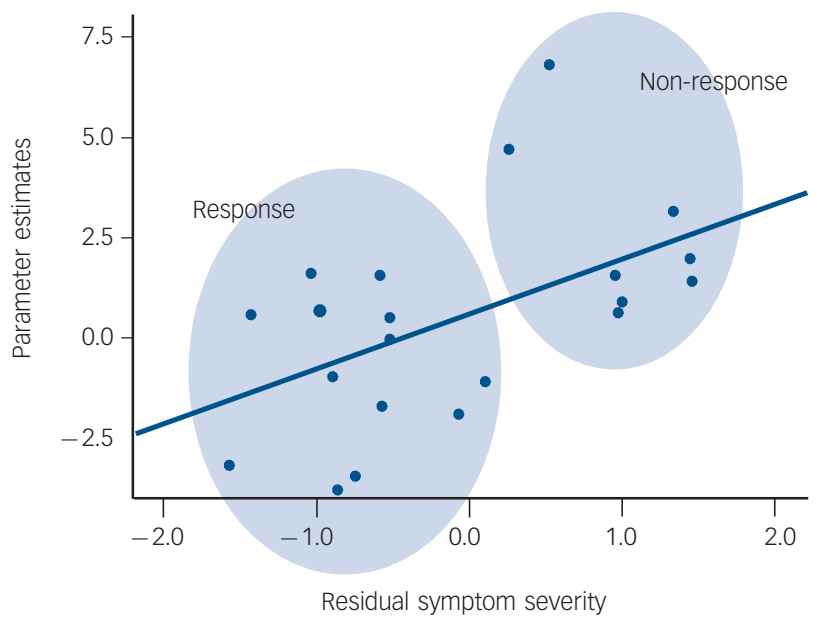

Fig. 2 Right amygdala activity. (a) Enhanced activity in the right amygdala in the non-responder group compared with those responding to therapy $(P<0.001, \kappa>10)$. (b) Plotted mean beta parameter estimates (eigenvariates) and standard errors of contrast images (self-critical $v$. neutral) in the responder group (blue) and the non-responder group (white). (c) Relationship between right amygdala activity (beta parameter estimates) and z-standardised residual gain scores on the Beck Depression Inventory post-treatment.

\begin{tabular}{|c|c|c|c|c|c|c|c|c|}
\hline \multirow[b]{2}{*}{$\begin{array}{l}\text { Independent } \\
\text { variable }^{a}\end{array}$} & \multirow[b]{2}{*}{$\begin{array}{c}\text { Mediating } \\
\text { variable }^{\mathrm{b}}\end{array}$} & \multirow[b]{2}{*}{$\begin{array}{c}\text { Dependent } \\
\text { variable }^{\mathrm{b}}\end{array}$} & \multicolumn{5}{|c|}{ Standardised regression coefficient $(\beta)$} & \multirow[b]{2}{*}{$n$} \\
\hline & & & $\begin{array}{l}\text { Effect of IV } \\
\text { on MV (a) }\end{array}$ & $\begin{array}{c}\text { Effect of MV } \\
\text { on DV }(b)\end{array}$ & $\begin{array}{c}\text { Direct } \\
\text { effect }\left(c^{\prime}\right)\end{array}$ & $\begin{array}{l}\text { Indirect } \\
\text { effect }(a b)\end{array}$ & $\begin{array}{c}\text { Total } \\
\text { effect }(c)\end{array}$ & \\
\hline Amygdala activity & ERSQ & BDI-II post & $-0.54^{* *}$ & $-0.56^{*}$ & 0.19 & $0.30^{c}$ & $0.52^{*}$ & 21 \\
\hline Amygdala activity & ERSQ & BDI-II follow-up & $-0.55^{\star}$ & $-0.62^{* *}$ & 0.16 & $0.34^{\mathrm{C}}$ & $0.53^{*}$ & 18 \\
\hline Amygdala activity & DAS & BDI-II post & -0.01 & $0.61 * *$ & $0.51 * *$ & -0.006 & $0.50 *$ & 20 \\
\hline Amygdala activity & DAS & BDI-II follow-up & 0.10 & 0.35 & 0.44 & 0.04 & 0.50 & 17 \\
\hline \multicolumn{9}{|c|}{ 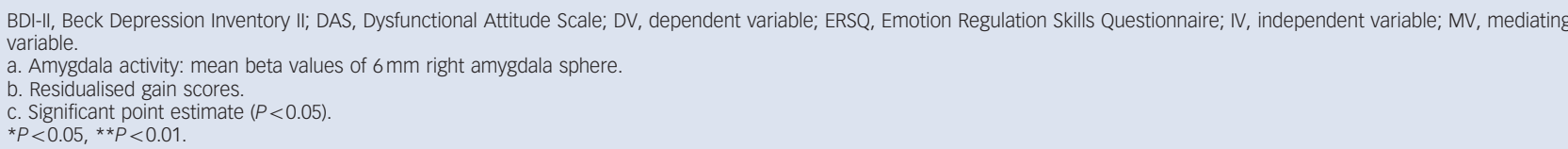 } \\
\hline
\end{tabular}

responses in people with an MDE. At the cortical level we found enhanced activity in patients with an MDE in a distributed network of the bilateral occipital cortex, mainly in higher-order visual areas, extending to the bilateral fusiform gyrus. In the left hemisphere, activations expanded into the temporal cortex. Converging evidence from both animal and human research suggests emotion- and attention-related modulation of visual processing. Emotional induction using visual stimuli probably activates the occipital cortex and enhanced activity in these regions might be driven by hyperactivity of the amygdala, which has strong anatomical connections to visual areas. ${ }^{33,34}$

\section{Response $\boldsymbol{V}$. non-response}

Participants who did not respond to therapy showed increased neural activity in the right amygdala during confrontation with self-critical material when compared with those who did respond. In addition, brain activity in this region correlated negatively with 
CBT outcome. These results indicate that there might be a subgroup of patients with MDE with enhanced amygdala activity in response to emotional stimuli, predicting poorer CBT outcome. Ruling out alternative explanations of the observed differences between the responder and non-responder groups, these groups did not differ in terms of gender distribution, age, marital status, highest educational level, single or recurrent episode, chronicity, comorbid anxiety disorder or baseline symptom severity. The last suggests that amygdala hyperactivity may be more than a marker of severe depression. Therefore, future research should examine the risk factors of insufficient treatment response such as depression subtypes, personality factors or genetics that are associated with elevated amygdala response in more detail. Remarkably, Bryant et al reported a similar association between amygdala activity at baseline and insufficient treatment response in patients with PTSD, ${ }^{17}$ raising the question whether amygdala hyperreactivity may be a transdiagnostic risk factor for therapy non-response. In support of this notion, a recent meta-analysis reported an association between increased activation in the right amygdala, striatum and insula and an increased likelihood of poor response to antidepressant medication. ${ }^{16}$

\section{Mechanisms of change}

Mediation analyses showed that the negative relationship between right amygdala activation and change in depressive symptoms during therapy might be explained by improvement of emotion regulation skills. Given that there was no mediation for change in dysfunctional attitudes, the specificity of this finding for emotional changes is highlighted. ${ }^{35}$ Considering these results, we propose that high levels of amygdala activation by individualised self-critical stimuli at baseline may hamper patients' readiness to learn new emotion regulation skills. In support of this hypothesis, a recent study demonstrated that adding emotion regulation training to CBT for depression given as an in-patient treatment improved treatment outcome. ${ }^{36}$

\section{Study implications and limitations}

To our knowledge this is the first study to examine the potential change mechanisms underlying a biomarker-outcome association. At this point it would be premature to derive clinical consequences from these results, as replication of the findings will be necessary. However, the findings may add to the quest for tailored treatment strategies by uncovering a subgroup of patients with depression with amygdala hyperactivity possibly benefiting from initial training in emotion regulation skills before engaging in emotionally challenging interventions in the course of psychotherapy. As an alternative to compensating for skill deficits, therapists might additionally search for individual strengths to build on when dealing with challenging emotions, such as memories of previously mastered challenges or interpersonal resources. Along these lines, a study has reported better outcomes in personalising treatment to patients' relative strengths than to their relative deficits. ${ }^{37}$ Whereas our study identified a possible link between neural activity at baseline and a psychological mechanism of change, it is still unclear exactly how amygdala hyperactivity might impede the development of emotion regulation skills. One explanation might be that overly strong emotions are experienced as disturbing and overwhelming, and consequent avoidance of displeasing emotions might impede or even prevent emotional change. In any case, before deducing practical implications it would be necessary to gain more information about factors potentially interconnecting amygdala hyperreactivity, impeded development of emotional skills and treatment outcome.
A main limitation of our study was the lack of a no-treatment control group. As a consequence, we were not able to exclude the possibility that the observed correlation of amygdala hyperactivity and non-response might have resulted from more general effects occurring in psychotherapy as well as in drug treatment, such as time- and/or placebo effects. Another important limitation of the study is its small sample size; our results must therefore be regarded as strictly preliminary. An additional limitation may be that we did not systematically assess negativity ratings of the neutral words during or after the scan. Given the negativity bias of people with depression, this might have had an influence on the self-critical $v$. neutral contrast that we could not control. Optimally, negativity ratings should be assessed immediately after presentation during the scan. However, as this would imply introducing a significant cognitive component potentially interfering and decreasing activation in limbic regions, we refrained from this option. Future studies might assess negativity bias using post-scan ratings. Methodologically, we recommend the use of in-session assessments of emotion processing and emotional change in psychotherapy to clarify further the associations between process-outcome relationships and neural predictors. Nevertheless, this interdisciplinary research strategy holds great promise for tailoring the treatment of patients with depression in the service of better outcomes.

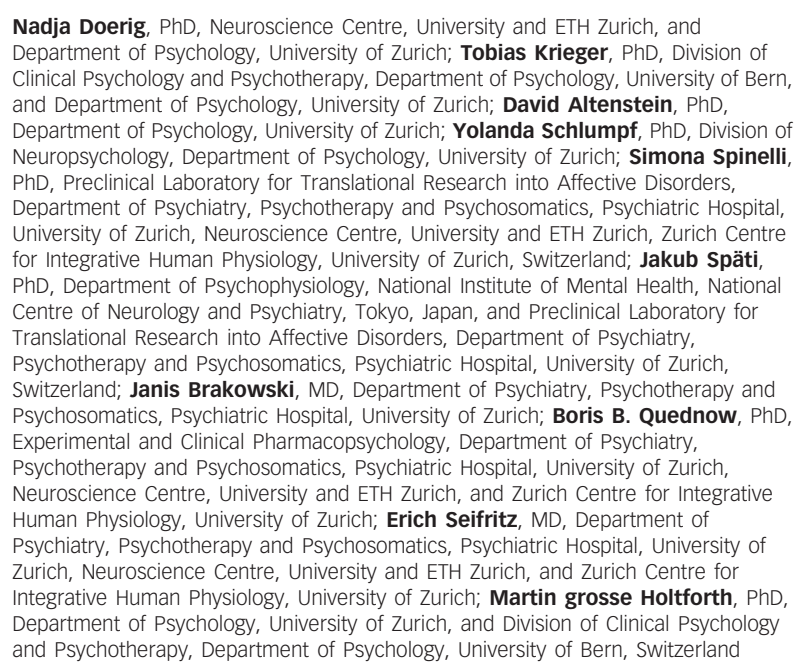

Nadja Doerig, PhD, Neuroscience Centre, University and ETH Zurich, and Department of Psychology, University of Zurich; Tobias Krieger, PhD, Division of Clinical Psychology and Psychotherapy, Department of Psychology, University of Bern and Department of Psychology, University of Zurich; David Altenstein, PhD, Department of Psychology, University of Zurich; Yolanda Schlumpf, PhD, Division of Neuropsychology, Department of Psychology, University of Zurich; Simona Spinelli, $\mathrm{PhD}$, Preclinical Laboratory for Translational Research into Affective Disorders, Department of Psychiatry, Psychotherapy and Psychosomatics, Psychiatric Hospital, University of Zurich, Neuroscience Centre, University and ETH Zurich, Zurich Centre for Integrative Human Physiology, University of Zurich, Switzerland; Jakub Späti, $\mathrm{PhD}$, Department of Psychophysiology, National Institute of Mental Health, Nationa Centre of Neurology and Psychiatry, Tokyo, Japan, and Preclinical Laboratory for Translational Research into Affective Disorders, Department of Psychiatry, Psychotherapy and Psychosomatics, Psychiatric Hospital, University of Zurich, Switzerland; Janis Brakowski, MD, Department of Psychiatry, Psychotherapy and Psychosomatics, Psychiatric Hospital, University of Zurich; Boris B. Quednow, PhD Experimental and Clinical Pharmacopsychology, Department of Psychiatry, Psychotherapy and Psychosomatics, Psychiatric Hospital, University of Zurich, Neuroscience Centre, University and ETH Zurich, and Zurich Centre for Integrative Human Physiology, University of Zurich; Erich Seifritz, MD, Department of Psychiatry, Psychotherapy and Psychosomatics, Psychiatric Hospital, University of Zurich, Neuroscience Centre, University and ETH Zurich, and Zurich Centre for Integrative Human Physiology, University of Zurich; Martin grosse Holtforth, PhD Department of Psychology, University of Zurich, and Division of Clinical Psychology and Psychotherapy, Department of Psychology, University of Bern, Switzerland

Correspondence: Nadja Doerig, Binzmuehlestrasse 14/19, 8050 Zurich Switzerland. Email: nadja.doerig@psychologie.uzh.ch

First received 14 Apr 2014, final revision 9 Nov 2014, accepted 12 Dec 2014

\section{Funding}

This research was supported by the Swiss National Foundation (grant PP00P1-123377/1 to M.G.H. and grant PZOOP3-126363 to S.S.) as well as a research grant by the Foundation for Research in Science and the Humanities at the University of Zurich to M.G.H. and the Clinical Research Priority Program Molecular Imaging at the University of Zurich to ES. B.B.Q. was subsidised by grants from the Swiss National Science Foundation (grant PPOOP1-12351611 and PPOOP1-14632611).

\section{Acknowledgements}

The authors would like to thank all the therapists and supervisors for their outstanding work delivering the treatment. We further thank our research assistants L. Meisch, MSc, H. Hermann, MSC, and H. Ospelt, BSc, for their tireless efforts in running the clinical trial, as well as collecting the trial data. We further thank Dr P. Staempfli and Dr E. Sydekum for their valuable help with study procedures and P. Kausch, MSC, and H. Ospelt, MSC, for their help with functional magnetic resonance imaging data collection. Most importantly, we thank all the people who participated in the research and gave so generously of their time in data collection and providing feedback about the study. 


\section{References}

1 Zuroff DC, Igreja I, Mongrain M. Dysfunctional attitudes, dependency, and self-criticism as predictors of depressive mood states: a 12-month longitudinal study. Cognit Ther Res 1990; 14: 315-26.

2 Beck AT, Rush AJ, Shaw BF, Emery G. Cognitive Therapy of Depression. Guilford, 1979

3 Drevets WC, Price LP, Furey ML. Brain structural and functional abnormalities in mood disorders: implications for neurocircuitry models of depression. Brain Struct Funct 2008; 213: 93-118.

4 Suslow T, Konrad C, Kugel H, Rumstadt D, Zwitserlood P, Schoning S, et al. Automatic mood-congruent amygdala responses to masked facial expressions in major depression. Biol Psychiatry 2010; 67: 155-60.

5 Anand A, Li Y, Wang Y, Wu J, Gao S, Bukhari L, et al. Activity and connectivity of brain mood regulating circuit in depression: a functional magnetic resonance study. Biol Psychiatry 2005; 57: 1079-88.

6 Phillips ML, Drevets WC, Rauch SL, Lane R. Neurobiology of emotion perception I: the neural basis of normal emotion perception. Biol Psychiatry 2003; 54: 504-14.

7 Sheline YI, Barch DM, Donnelly JM, Ollinger JM, Snyder AZ, Mintun MA Increased amygdala response to masked emotional faces in depressed subjects resolves with antidepressant treatment: an fMRI study. Biol Psychiatry 2001; 50: 651-8.

8 Kessler H, Taubner S, Buchheim A, Munte TF, Stasch M, Kachele H, et al. Individualized and clinically derived stimuli activate limbic structures in depression: an fMRI study. PLOS ONE 2011; 6: e15712.

9 Fitzgerald PB, Laird AR, Maller J, Daskalakis ZJ. A meta-analytic study of changes in brain activation in depression. Hum Brain Mapp 2008; 29: 683-95.

10 Townsend JD, Eberhart NK, Bookheimer SY, Eisenberger NI, Foland-Ross LC Cook IA, et al. FMRI activation in the amygdala and the orbitofrontal cortex in unmedicated subjects with major depressive disorder. Psychiatry Res 2010; 183: 209-17.

11 Siegle GJ, Thompson W, Carter CS, Steinhauer SR, Thase ME. Increased amygdala and decreased dorsolateral prefrontal BOLD responses in unipolar depression: related and independent features. Biol Psychiatry 2007; 61: 198-209.

12 Siegle GJ, Steinhauer SR, Thase ME, Stenger VA, Carter CS. Can't shake that feeling: event-related fMRI assessment of sustained amygdala activity in response to emotional information in depressed individuals. Biol Psychiatry 2002; 51: 693-707.

13 Hooley JM, Gruber SA, Parker HA, Guillaumot J, Rogowska J, Yurgelun-Todd DA. Cortico-limbic response to personally challenging emotional stimuli after complete recovery from depression. Psychiatry Res 2009; 172: 83-91.

14 Costafreda SG, Brammer MJ, David AS, Fu CH. Predictors of amygdala activation during the processing of emotional stimuli: a meta-analysis of 385 PET and fMRI studies. Brain Res Rev 2008; 58: 57-70.

15 Doerig N, Schlumpf Y, Spinelli S, Spati J, Brakowski J, Quednow BB, et al. Neural representation and clinically relevant moderators of individualised self-criticism in healthy subjects. Soc Cogn Affect Neurosci 2014; 9: 1333-40.

$16 \mathrm{Fu} \mathrm{CH}$, Steiner H, Costafreda SG. Predictive neural biomarkers of clinical response in depression: a meta-analysis of functional and structural neuroimaging studies of pharmacological and psychological therapies. Neurobiol Dis 2013; 52: 75-83.

17 Bryant RA, Felmingham K, Kemp A, Das P, Hughes G, Peduto A, et al. Amygdala and ventral anterior cingulate activation predicts treatment response to cognitive behaviour therapy for post-traumatic stress disorder. Psychol Med 2008; 38: 555-61.

18 Hollon SD, Munoz RF, Barlow DH, Beardslee WR, Bell CC, Bernal G, et al. Psychosocial intervention development for the prevention and treatment of depression: promoting innovation and increasing access. Biol Psychiatry 2002; 52: 610-30.

19 Furlong M, Oei TP. Changes to automatic thoughts and dysfunctional attitudes in group CBT for depression. Behav Cogn Psychother 2002; 30 351-60.

20 Fehlinger T, Stumpenhorst M, Stenzel N, Rief W. Emotion regulation is the essential skill for improving depressive symptoms. J Affect Disord 2012; 144: 116-22.

21 Hautzinger M, Keller F, Kuhner C. Das Beck Depressionsinventar II. Deutsche Bearbeitung und Handbuch zum BDI II [Beck Depression Inventory II]. Harcourt Test Services, 2006.

22 Wittchen HU, Zaudig M, Fydrich T. Strukturiertes Klinisches Interview für DSM-IV [Structured Clinical Interview for DSM-IV (German version)]. Hogrefe, 1997.

23 Kuhner C, Burger F, Keller M, Hautzinger M. Reliability and validity of the Revised Beck Depression Inventory (BDI-II). Nervenarzt 2007; 78: 651-6.

24 Berking $M$, Znoj HJ. Entwicklung und Validierung eines Fragebogens zur standardisierten Selbsteinschätzung emotionaler Kompetenzen (SEK-27). [Development and validation of a self-report measure for the assessment of emotion regulation skills (SEK-27).] Z Psychiatr, Psychol Psychother 2008; 56: 141-53.

25 Hautzinger M, Luka U, Trautmann RD. Skala dysfunktionaler Einstellungen-Eine deutsche Version der Dysfunctional Attitude Scale. [Dysfunctional Attitude Scale (German version).] Diagnostica 1985; 31: 312-23.

26 Laux L, Glanzmann P, Schaffner CD, Spielberger D. Das State-TraitAngstinventar [State-Trait-Anxiety Inventory (STAl) (German version).]. Beltz, 1981.

27 Riedel M, Moller HJ, Obermeier M, Schennach-Wolff R, Bauer M, Adli M, et al. Response and remission criteria in major depression - a validation of current practice. J Psychiatr Res 2010; 44: 1063-8.

28 Pruessmann KP, Weiger M, Scheidegger MB, Boesiger P. SENSE: sensitivity encoding for fast MRI. Magn Reson Med 1999; 42: 952-62.

29 Friston KJ, Ashburner JT, Kiebel SJ, Nichols TE, Penny WD. Statistical Parametric Mapping: The Analysis of Functional Brain Images. Academic Press, 2011.

30 Preacher KJ, Hayes AF. SPSS and SAS procedures for estimating indirect effects in simple mediation models. Behav Res Methods 2004; 36: 717-31.

31 Drevets WC, Videen TO, Price JL, Preskorn SH, Carmichael ST, Raichle ME. A functional anatomical study of unipolar depression. J Neurosci 1992; 12 3628-41.

32 Stein MB, Simmons AN, Feinstein JS, Paulus MP. Increased amygdala and insula activation during emotion processing in anxiety-prone subjects. Am J Psychiatry 2007; 164: 318-27.

33 Amaral $D$, Behniea $H$, Kelly J. Topographic organization of projections from the amygdala to the visual cortex in the macaque monkey. Neuroscience 2003; 118: 1099-120.

34 Vuilleumier $\mathrm{P}$, Driver J. Modulation of visual processing by attention and emotion: windows on causal interactions between human brain regions. Philos Trans R Soc Lond B Biol Sci 2007; 362: 837-55.

35 Kazdin A. Mediators and mechanisms of change in psychotherapy research. Annu Rev Clin Psychol 2007; 3: 1-27.

36 Berking M, Ebert D, Cuijpers P, Hofmann SG. Emotion regulation skills training enhances the efficacy of inpatient cognitive behavioral therapy for major depressive disorder: a randomized controlled trial. Psychother Psychosom 2013; 82: 234-45.

37 Cheavens JS, Strunk DR, Lazarus SA, Goldstein LA. The compensation and capitalization models: a test of two approaches to individualizing the treatment of depression. Behav Res Ther 2012; 50: 699-706. 